\title{
Leptina: más que una adipocina, una herramienta para la comprensión de la obesidad y el riesgo cardiovascular
}

\author{
Ángela Gómez ${ }^{1}$, Jorge Palacio ${ }^{2}$, Adriana Jaramillo ${ }^{3}$ Ricardo Rosero ${ }^{4 *}$
}

Clínica de Obesidad, Dismetabolismo y Deporte, División de Endocrinología, Departamento de Medicina Interna, Clínica Las Américas, Medellín, Colombia.

${ }^{1}$ Médica Residente de Pediatría, Universidad de la Sabana, Chía

${ }^{2}$ Médico Deportólogo, COD2, Clínica Las Américas, Medellín, Colombia.

${ }^{3}$ Médica Pediatra y Endocrinóloga, COD2, Clínica Las Américas, Medellín, Colombia.

${ }^{4}$ Médico Internista y Endocrinólogo, COD2, Clínica Las Américas, Medellín, Colombia.

* Correspondencia: rosero.ravelo@gmail.com

Fecha de recepción: 30/01/2018

Fecha de aceptación: 1/03/2018

\section{Resumen}

$\mathrm{L}$ a obesidad es considerada actualmente como la epidemia del siglo XXI. El acelerado aumento de la prevalencia y mortalidad a causa de enfermedades cardiovasculares establece un precedente histórico como problema de salud pública mundial. El aumento en la incidencia de obesidad y enfermedades crónicas (enfermedad coronaria, cáncer, diabetes), así como la frecuente consulta por este problema en la práctica clínica, han llevado a profundizar en el entendimiento del eslabón que las une. Existen numerosos factores neuroendocrinos responsables de la regulación del metabolismo energético. Sin embargo, la leptina es quizás el mediador más estudiado de este balance. Se ha asociado a vías de señalización que son comunes en estados proinflamatorios como la resistencia a la insulina en tejido adiposo y la ateromatosis a nivel de endotelio. Factores ambientales como el envejecimiento, las alteraciones en el sueño y trastornos psiquiátricos se relacionan con este "epifenómeno", los cuales, a través de su efecto sobre el estado inflamatorio de la obesidad, repercuten en la homeostasis energética y el mantenimiento del peso corporal normal con impacto consecuente en el estado cardiometabólico del individuo según lo señalan numerosos estudios. El descubrimiento de nuevas vías de comunicación, la acción de citocinas y adipocinas en común y la redefinición del concepto de obesidad plantean un panorama promisorio hacia nuevos objetivos de riesgo cardiovascular asociados a obesidad.

En este artículo se hará una revisión del papel de la leptina en la enfermedad cardiovascular y la obesidad a través de los mecanismos fisiopatológicos que comparten, con miras hacia la identificación temprana de riesgo cardiometabólico y el impacto en morbimortalidad asociada a adiposidad.

Palabras clave: leptina, obesidad, enfermedad cardiovascular, riesgo cardiometabólico.

\section{Introducción}

El concepto de obesidad ha cambiado dramáticamente en el último siglo, pasando de ser un factor de riesgo caracterizado por acumulación de tejido graso quiescente ${ }^{(1)}$ a una enfermedad crónica multisistémica, asociada a un disbalance positivo entre consumo energético y gasto calórico, con efectos deletéreos en la salud ${ }^{(2,3)}$.

La obesidad es un estado proinflamatorio en el que entran a jugar factores ambientales, neuroendocrinos y genéticos ${ }^{(3)}$. Durante las últimas décadas, el aumento del consumo per cápita de comidas procesadas y la cantidad en las porciones ${ }^{(4)}$, el sedentarismo favorecido por el uso de aparatos electrónicos, la reducción de la actividad física ${ }^{(5,6)}$ y alteraciones del sueño ${ }^{(7)}$, han mostrado una relación directa con el aumento de la prevalencia de esta enfermedad ${ }^{(8)}$.

Se han identificado mediadores implicados y su relación con otras enfermedades. Entre los más estudiados están la leptina, adiponectina, TNF alfa, interleucinas 1 y 6 (IL-1, IL-6), principalmente descritas también en el síndrome metabólico ${ }^{(9)}$. Sin embargo, se destaca la leptina por ser uno de los productos principales del tejido graso, el cual ha servido como desencadenante de investigaciones alrededor de la homeostasis energética corporal y el desarrollo de la obesidad ${ }^{(3)}$. La leptina tiene un efecto multiorgánico: en hipotálamo tiene una acción anorexígena $^{(10)}$ y a nivel óseo, favorece la densidad y, la mineralización ósea ${ }^{(11)}$. En fases de normalidad, promueve además el consumo de ácidos grasos por parte del músculo esquelético, generando posteriormente resistencia a la insulina ${ }^{(12)}$. De igual forma, en la relación entre leptina y daño articular se ha descrito aumento de niveles de ésta, del TNF alfa y de IL proinflamatorias en la grasa de Hoffa en pacientes con altos índices grasos ${ }^{(13)}$. El endotelio contribuye al proceso de ateromatosis, dando origen a la relación con el desarrollo de enfermedades crónicas, hipertensión arterial y enfermedad coronaria ${ }^{(9,10,14)}$.

Existen estudios que describen la relación entre leptina y mortalidad cardiovascular en sujetos adultos ${ }^{(15,16)}$. En el caso 
del estudio NAHNES III en el 2015, el cual siguió 1.794 participantes a 12 años y describió la leptina como marcador de riesgo de mortalidad cardiovascular en individuos mayores de 60 años; con base en el concepto de obesidad que involucra además la definición de índice de masa corporal (IMC) $\geq 30 \mathrm{~kg} /$ $\mathrm{m}^{2}$, porcentaje de grasa corporal $\geq 25 \%$ en hombres $\mathrm{y} \geq 35 \%$ en mujeres, circunferencia abdominal $\geq 102 \mathrm{~cm}$ en hombres $y \geq 88 \mathrm{~cm}$ en mujeres (aunque con diversidad de conceptos cuando se homologa a Federación Internacional de Diabetes con medidas de $90 \mathrm{~cm}$ en hombres y $80 \mathrm{~cm}$ en mujeres); y relación cintura-cadera $\geq 0,85$ en mujeres y 0,95 o más en hombres $^{(17)}$. Todo lo anterior se debe considerar como los factores comunes en la obesidad y sus comorbilidades.

\section{Leptina, el "adipostato"}

La leptina es una adipocina cuyo nombre procede del griego "leptos" que significa delgado. Se deriva del tejido graso blanco principalmente ${ }^{(3)} \mathrm{y}$ en menor proporción de placenta, glándula mamaria, médula ósea, tejido linfoide y ovarios ${ }^{(18)}$. Fue descubierta en 1950 por Zhang et al., en la universidad de Rockefeller, a partir del hallazgo de roedores con características de hiperfagia, obesidad, letargia e hiperglucemia moderada, cepa que se designó con el nombre de ob/ob ${ }^{(19)}$ y que dio origen al denominado "gen de la obesidad". Bioquímicamente, la leptina es un péptido de 167 aminoácidos ${ }^{(20)}$, también conocido como "el adipostato" por su acción supresora del apetito y función neuroendocrina del control del peso corporal a través de la regulación de ingesta, gasto calórico y almacenamiento de tejido graso ${ }^{(21)}$. Actúa como marcador de las reservas energéticas del organismo y se le atribuyen además funciones a nivel reproductor e inmune ${ }^{(3,22)}$.

La leptina tiene una secreción pulsátil y sigue el ciclo circadiano, con mayor amplitud de onda en sujetos obesos y en consecuencia con niveles mayores circulantes en sangre $(\geq 15$ $\mathrm{ng} / \mathrm{mL}$ ) frente a personas con IMC normal (1 a $15 \mathrm{ng} / \mathrm{mL}$ ). Es transportada parcialmente unida a proteínas plasmáticas y su eliminación es renal ${ }^{(3)}$.

La leptina producida en tejido adiposo, una vez liberada al torrente sanguíneo, actúa a través de la activación de su receptor (ObR) ubicado en sistema nervioso central en el hipotálamo (especialmente, núcleo arcuato) y a nivel periférico. Los receptores poseen al menos 6 isoformas, que se encargan del transporte de la leptina (ObRa,ObRb y ObRc) y de la señalización (ObRd, ObRe y ObRf) ${ }^{(23)}$. Inicialmente se encontró que sujetos obesos presentaban concentraciones séricas elevadas de leptina ${ }^{(24)}$, lo que planteó el estudio de la "resistencia a la insulina" como origen y/o mantenimiento de la obesidad. Posteriormente, se encontró que tal hallazgo era completamente acertado en aquellos con deficiencia congénita de leptina ${ }^{(24)}$. No obstante, cada día se vislumbran nuevos mecanismos que contribuyen a sugerir la resistencia a la leptina, como causa de obesidad.

\section{Fisiopatogenia}

La homeostasis energética corporal se da por un sistema complejo de procesos bioquímicos, ambientales y genéticos ${ }^{(8)}$, alcanzando un porcentaje de herencia en IMC del 40\%-70\% ${ }^{(25)}$. La regulación se inicia a partir de un órgano efector (tejido adiposo), que favorece la emisión de señales aferentes (leptina, entre otros) con acción en un centro regulador a nivel de sistema nervioso central (hipotálamo), en donde se integra el mensaje y señales eferentes que generan una respuesta a nivel periférico ${ }^{(3)}$.

Para comprender la fisiopatogenia de la obesidad debemos tomar el tejido adiposo como un compartimento corporal metabólicamente activo, cuyas características (distribución, tamaño y aporte sanguíneo), en el tiempo alteran otros órganos y tejidos como hígado, músculo esquelético, endotelio, etc ${ }^{(26)}$.

La influencia de las células adiposas sobre los tejidos, pasando por localización anatómica y la producción de citocinas y metabolitos por mecanismos paracrinos y endocrinos ${ }^{(27)}$ se hace relevante, ya que determinará los efectos metabólicos.

El tejido adiposo humano adulto está conformado por depósito de adipocitos predominantemente blancos (tejido graso blanco), ubicados en su gran mayoría en el tejido celular subcutáneo que aumentan en número y se redistribuyen de manera lenta en el desarrollo de la obesidad ${ }^{(26)}$. Su función fundamentalmente es de almacenamiento energético como reserva en caso de aumento de la demanda metabólica ${ }^{(28)}$. La mayor cantidad de energía es almacenada en forma de triglicéridos y colesterol, los cuales se asocian a producción de leptina y adiponectina ${ }^{(29)}$. En la obesidad, el exceso crónico en forma de lípidos (triglicéridos específicamente) provoca la redistribución de adipocitos, produciendo cambios proinflamatorios evidenciados por incremento de IL-1, IL-6 y macrófagos secundarios a apoptosis de adipocitos, con repercusión directa en el riesgo metabólico y cardiovascular ${ }^{(27,30)}$. Un claro ejemplo es el efecto de la grasa visceral (mesentérica y omental) vinculado a alteraciones metabólicas asociadas a la obesidad, como la diabetes mellitus tipo $2^{(31,32)}$. Paralelamente, produce aumento del gasto calórico basal, la presión arterial y la masa pancreática ${ }^{(26,33)}$, reflejándose en insulina y glucemia poscarga elevadas, que aumentan de manera lineal con el $\operatorname{IMC}^{(34)}$ lo que soporta la asociación con enfermedad coronaria y diabetes mellitus tipo $2^{(23)}$.

Otro tipo de tejido adiposo es el tejido graso pardo, descrito inicialmente en mamíferos menores como método de protección contra el frío o en periodos de hibernación. En humanos se considera en recién nacidos como elemento primordial frente a la termorregulación en el periodo perinatal ${ }^{(35)}$. Su proporción es muy inferior con respecto al tejido graso blanco, sin embargo, es posible encontrarla en grasa supraclavicular, paravertebral, mediastinal ${ }^{(36)} \mathrm{y}$, recientemente, estudios sugieren la presencia en tejido adiposo perivascular en ratones ${ }^{(37)}$.

El interés de su estudio radica en la producción de proteínas con efecto sobre la activación de termogénesis. La UCP-1 
(Uncoupling protein-1 o "proteína desacopladora"), también conocida como termogenina, actúa como vía alternativa a través de la cual los protones expulsados al espacio intermembranal a lo largo de la cadena respiratoria pueden retornar la matriz mitocondrial, permitiendo la disipación en forma de calor, de la energía almacenada por el bombeo de protones. La termogenina, como la ATP sintasa, actúa como un canal de protones localizado en la membrana mitocondrial interna que permite su translocación desde el espacio intermembranal mitocondrial hacia la matriz mitocondrial. Realiza, como su nombre lo indica, el desacople de la cadena productora de electrones que hace que la energía encargada de producir ATP se use en cambio en la producción de calorías. El coactivador de receptor gamma activado por proliferador de peroxisoma (PGC-1alfa) contribuye al incremento de la actividad mitocondrial del tejido adiposo pardo ${ }^{(38,39)}$.

El tejido graso beige es considerado la célula progenitora, la cual comparte características de ambos linajes blanco y pardo ${ }^{(37)}$. Sufre un proceso de transformación a partir del adipocito blanco, conocido como "apardamiento" (por su denominación en inglés "browning"), inducido por exposición al frío, ejercicio y estímulos hormonales específicos ${ }^{(8)}$. El tejido graso perivascular presenta características diferentes según su localización, comportándose como fenotipo de tejido adiposo blanco, y se comporta favoreciendo la inflamación a nivel de la aorta abdominal y es termogénicamente activo en la aorta torácica ${ }^{(40)}$. Hacen falta estudios que soporten y amplíen este concepto en humanos, ya que la caracterización del tejido adiposo perivascular podría traer aportes significativos en el campo de la protección metabólica y cardiovascular.

\section{Leptina y efecto cardiovascular}

La alteración de este balance como en cualquier órgano de la economía corporal, genera un proceso inflamatorio en respuesta a una noxa. El dinamismo de esta condición, como consecuencia de la permanente interacción con el ambiente, convierte la obesidad en una patología compleja de controlar. En un estudio hecho en roedores se los sometió a periodos de exceso de ingesta hipercalórica y ayuno, demostrando la capacidad celular de los adipocitos para aumentar hasta en 4 veces su tamaño o reducirlo dramáticamente en 24 horas de acuerdo con la demanda metabólica, estableciendo una plasticidad energética $^{(41)}$. La disfunción del tejido adiposo es de los principales factores que contribuyen al desarrollo de la obesidad con papel activo de células del sistema inmune innato y adaptativo ${ }^{(42,43)}$.

Las acciones de adipocinas, fibroblastos, células endoteliales y mediadores inmunológicos se generan en estados de hipoxia ante el incremento de lípidos, que favorece el aumento de tamaño adipocitario, con angiogénesis y matriz extracelular como mecanismo compensatorio agudo $^{(44)}$. Sin embargo, en casos en que la respuesta la hipoxia no es suficiente, apare- ce la llamada "triada ominosa", caracterizada por inflamación no resuelta, fibrosis y angiogénesis inadecuada ${ }^{(41)}$. Este estado resulta nocivo en condiciones crónicas de malnutrición, generando cambios conformacionales y funcionales en el microambiente adiposo en el que la expansión de los depósitos lipídicos supera la capacidad tisular para mantener adecuada oxigenación del mismo, generando alteración de la angiogénesis con posterior hipoxia persistente, fibrosis y muerte celular. Estas características de expansión anómala en tejido adiposo contribuyen a alteraciones metabólicas observadas en la obesidad y la diabetes mellitus tipo $2^{(41)}$. Así, la disfunción del tejido adiposo corresponde al punto de quiebre entre la respuesta temprana y autolimitada ante el incremento de aporte calórico y la perpetuación del proceso inflamatorio per se. Las elevadas concentraciones de ácidos grasos libres, particularmente triglicéridos, secundarias al aumento de hidrólisis, desencadenan la elevación de LDL y disminución de HDL, dando lugar a dislipidemia y lipotoxicidad hepática ${ }^{(45)}$. El aumento en la síntesis de adipocinas conlleva a disfunción en la señalización de insulina, generando hiperinsulinemia y estado de resistencia a la insulina, estrechamente asociado a diabetes mellitus tipo $2^{(36,46)}$.

La hiperreactividad crónica del sistema nervioso simpático, así como la activación del eje reina-angiotensina-aldosterona que podría estar asociado a los mecanismos fisiopatológicos de la hipertensión arterial en pacientes con obesidad; de igual forma a nivel mecánico, la obesidad cumple un papel fundamental en factores de riesgo cardiovasculares. El tejido adiposo perirrenal también podría contribuir a la hipertensión observada con frecuencia en pacientes con obesidad ${ }^{(47)}$. El incremento de la grasa visceral ${ }^{(29,36)}$, al igual que a nivel hepático, el aumento de tamaño liposomal (esteatosis), favorecen la acumulación de lípidos en vacuolas y aumento de condiciones asociadas como hígado graso no alcohólico, esteatohepatitis y cirrosis $^{(48)}$.

\section{Leptina y factores ambientales}

Recientemente se ha descrito cómo el envejecimiento y las alteraciones del ciclo circadiano favorecen las rutas metabólicas de la obesidad, con especial interés en el efecto sobre la señalización de la leptina y la inducción de resistencia independientemente de otros factores de riesgo ${ }^{(49)}$. La vía más mencionada es la JACK2/STAT 3, cuya activación se inicia con los factores de transcripción, a través de procesos de fosforilación de proteínas pertenecientes a las familias STAT 3, ERK (cinasa estimulada por señales extracelulares) e IRS (insulin receptor subtrate ${ }^{(50)}$. Las dos primeras actúan en la transcripción genómica. La STAT 3 activa la propiomelanocortina (POMC), la cual libera sustancias anorexígenas (neuropéptido $Y)^{(51)}$. La alteración en esta vía da como resultado casos de hiperleptinemia, hiperfagia, ganancia de peso e hiperinsulinemia $^{(52)}$. 
La ERK, por su parte, regula la proliferación, diferenciación y supervivencia celular en condiciones normales, lo que representa un caso de alteración de leptina o resistencia a la leptina, fallo en transcripción y consecuente apoptosis celular ${ }^{(53)}$, estableciéndose como posible punto de relación entre obesidad y cáncer. La fosforilación de la IRS por JACK 2, da lugar a la activación de IP3K (fosfatidilinositol 3 cinasa), actuando en tejido adiposo, hígado, músculo y células cancerígenas. A nivel cerebral, produce pérdida de peso secundario al efecto anorexígeno, dando como resultado de su alteración, el aumento en el peso corporal por mecanismo similar al del STAT $3^{(54)}$. En 2015, Kettner el al. describieron cómo en roedores, el accionar del reloj biológico sobre la transcripción de la leptina en el tejido adiposo revela una relación directa ${ }^{(49)}$.

\section{Discusión}

Actualmente, el descubrimiento y entendimiento de nuevos conocimientos asociados a la obesidad nos ha abierto la oportunidad de comprender cómo este trastorno es una enfermedad de múltiples redes moleculares, hormonales e inclusive emocionales, que favorecen el desequilibrio de sus componentes principales, el que podríamos llamar, emulando los principios de la fisiopatología de la diabetes, como el "triunvirato de la obesidad"; en él convergen tres integrantes claves en el entendimiento y manejo de esta patología, los cuales son: el hipotálamo, el tejido adiposo (adipocito) y el tejido magro (miocito). En este punto, está anclada la relevancia de la leptina y la razón por la cual se decidió ver más allá de una adipocina, y considerar postularla junto con las curvas de glucosa e insulina en el perfil bioquímico adecuado para los pacientes dismetabólicos (preobesidad, obesidad y "sanos adiposos"), entendiendo que esta adipocina tiene relación y función asociadas directas e indirectas en los tres órganos claves del triunvirato; así pues, se logra entender el comportamiento alimentario desde la pérdida de la saciedad, asociado a la resistencia a la leptina, además de la hiperproducción de leptina que en niveles elevados se asocia a otras adipocinas inflamatorias. Spenedi et al., también lo mencionan en su escrito "La tormentosa relación de los ácidos grasos"(12). Así, inferimos la inflamación del paciente ("adipocitis") y en forma asociada podríamos abordar la elevación de la miostatina que inhibe la liberación de miocinas antiinflamatorias, empoderando al adipocito en el control metabólico de la obesidad y deteriorando el papel protector del músculo en el control de la enfermedad (hasta el nivel máximo conocido como obesidad sarcopénica).

Si somos coherentes en aceptar este concepto de modelo paraclínico del paciente dismetabólico o adipositario, podríamos excluir de nuestro pensamiento el concepto previo del "obeso sano", término que se ha postulado en referencia a un IMC elevado con perfil bioquímico aceptable; así entenderíamos que no se necesita tener un IMC elevado para estar en un ambiente hos- til que conlleva múltiples manifestaciones altamente mórbidas. Tal vez esta sería una herramienta útil para saber orientar la prevención de la enfermedad asociada a la corresponsabilidad del paciente en su manejo, y no solo basarse en el IMC elevado para su abordaje farmacológico. Lo último no excluye de la necesidad de intervenir al paciente con medicamentos, pero si podría dar más idea de nuestros objetivos primarios.

Debemos alejarnos del concepto inmediatista de la báscula, como único referente de mejoría del paciente y de que en el día a día nos estamos encontrando con mayor población con enfermedades establecidas no asociadas a IMC severamente elevados. Por el contrario, la mayor carga de la enfermedad está en los paciente con sobrepeso y obesidad grado ${ }^{(55)}$, entendiendo que el IMC es un buen punto de referencia en estudios poblacionales, mas no en la práctica diaria; esto porque perdemos de vista un gran número de pacientes con alto índice graso que ya tiene alteraciones en las vías proinflamatorias, que tal vez la leptina nos pueda enfocar. Así pues, el enfoque preventivo una vez más logramos volverlo "paliativo o crónico" como se ha venido haciendo en la mayoría de las enfermedades crónicas no transmisibles.

La estrecha relación entre la leptina y los mecanismos fisiopatológicos de riesgo cardiovascular propone un panorama hacia marcadores predictores de riesgo cardiometabólico, asociado a homeostasis de la glucosa/insulina y leptina que nos ayudan como herramienta de gestión del mismo riesgo; además de develar quién está dominando en la "balanza de tres puntos", para intentar establecer las diferentes estrategias de atención del paciente de forma individualizada, pues se ha demostrado que el abordaje independiente de cada una no genera grandes desenlaces.

\section{Conclusión}

La posible utilidad de la leptina como marcador bioquímico en la obesidad o adiposidad alterada radica en la identificación del proceso inflamatorio de la obesidad, que se asocia a un mayor riesgo de complicaciones cardiometabólicas (por ahora, la evidencia se asocia a población masculina mayor de 60 años), la cual debería complementarse con las curvas de insulina y glucosa para poder tener un abordaje claro y temprano del riesgo del paciente.

Aún no hay datos suficientemente precisos para tener que llevar control pos intervención, pero sí abre una brecha para establecer un perfil bioquímico de caracterización de los pacientes con índices grasos alterados, independientes del IMC.

Para finalizar, es muy importante tener claro que la obesidad es mucho más compleja que el número que arroje una báscula y su relación con la talla; por esto es trascendental buscar biomarcadores que permitan hacer la evaluación de riesgo cardiovascular temprano. Tal vez en mujeres hay adecuada evidencia del uso de leptina. 


\section{Agradecimientos}

Los autores agradecen a la Dirección Científica de la Clínica Las Américas por su colaboración en la revisión crítica y preparación del manuscrito

\section{Conflictos de interés}

RR: Ha recibido honorarios como conferencista para Novo
Nordisk y Pfizer, y es miembro del Advisory Board de Novo Nordisk.

JP: Ha recibido honorarios como conferencista para Novo Nordisk y MSD.

AG: No tiene conflictos que revelar.

Para la elaboración de este artículo no se recibió ningún patrocinio por parte de la industria farmacéutica.

\section{Referencias}

1. Popkin BM, Adair LS, Wen NgS. Now and then: The Global Nutrition Transition: The Pandemic of Obesity in Developing Countries. Nutr Rev. 2012 Jan; 70(1): 3-21.

2. Hall KD, Guo J, Dore M, Chow CC. The progressive increase of food waste in America and its environmental impact. PLoS One 2009; 4(11):E7940

3. Simón EL BAS. Leptina y obesidad. Anales del sistema sanitario de Navarra. 2002; 25:53-64.

4. Popkin BM, Hawkes C. Sweeting of the global diet, particularly beverag-es: patterns, trends, and policy responses. J. Diabetes Endocrinol 2016; 4:174-86.

5. Church TS1, Thomas DM, Tudor-Locke C, Katzmarzyk PT, Earnest CP, Rodarte RQ et.al. Trends over 5 decades in U.S. occupation-related physical activity and their associations with obesity. PLoS One 2011;6(5):e19657.

6. Von Loeffelholz C. The Role Non-Exercise Activity Thermogenesis in Human Obesity. In: De Groot LJ, ed. Endotext. South Darmouth, MA: MDText.com, 2000.

7. McAllister EJ, Dhurandhar NV, Keith SW, et al. Ten putative contributors to the obesity epidemic. Crit Rev Food Sci Nutr 2009; 49:868-913.

8. Heymsfiel SB, Wadden TA. Mechanisms, Pathophysiology, and Management of Obesity. N Engl J Med 2017; 376:254-66.

9. Akbaraly TN, Kivimaki M, Ancelin ML, et al. Metabolic syndrome,its components, and mortality in the elderly. J Clin Endocrinol Metab. 2010; 95(11):E327-E332.

10. Pasco JA, Henry MJ, Kotowicz MA, Collier GR, Ball MJ, Ugoni AM, et al. Serum leptin levels are associated with bone mass in nonobese women. J Clin Endocrinol Metab 2001;86(5):1884-7.

11. Rosero, R. Sierra A, Medina A. Interacción de la Leptina y hueso en sujetos obesos Protección vs Fragilidad. Rev Col Endo Dia y Metabol 2015; 2(3):14-21.

12. Costa Gil JR. Spinedi E. La tormentosa relación entre la grasa y el desarrollo de la diabetes mellitus de tipo 2: Rev Argent Endocrinol Metab. 2017;54(3):109-123.

13. Paduszyński W, Jeśkiewicz M, Uchański P, Gackowski S, Radkowski M, Demkow U, Hoffa's fat pad abnormality in the development of knee osterarthiritis. Adv Exp Med Biol. 2017 Aug 3. doi: 10.1007/5584_2017_77.

14. Health United States, 2015: with special feature on racial and ethnic health disparities. Hyattsville, MD: Centers for Disease Control and Prevention, National Center for Health Statistics, 2016:461.

15. Lieb W, Sullivan LM, Harris TB, et al. Plasma leptin levels and incidence of heart failure, cardiovascular disease, and total mortality in elderly individuals. Diabetes Care 2009; 32(4):612-616.

16. Welsh P, Murray HM, Buckley BM, et al. Leptin predicts diabetes but not cardiovascular disease: results from a large prospective study in an elderly population. Diabetes Care. 2009; 32(2):308-310.

17. Batsis JA, Sahakyan KR, Singh P, Bartels SJ, Somers VK. Leptin, Adiposity, and Mortality: Results from the National Health and Nutrition Examination Survey III, 1988 to 1994. Mayo Foundation for Medical Education and Research, Mayo Clin Proc. 2015; 90(4):481-491.

18. Dardeno TA, Chou SH, Moon HS, Chamberland JP, Fiorenza CG, Man-tzoros CS. Leptin in human physiology and therapeutics. Frontiers in neu-roendocrinology. 2010; 31(3):377-93.

19. Tartaglia LA, Dembski M, Weng X, Deng N, Culpepper J, Devos R, et al. Identification and expression cloning of a leptin receptor, OB-R. Cell. 1995; 83(7):1263-71.

20. Friedman JM. Leptin, leptin receptors, and the control of body weight. Na-ture. 1998 Oct 22;395(6704):763-70.

21. Mantzoros CS. The role of leptin in human obesity and disease: a review of current evidence. Ann Intern Med. 1999; 130(8):671-680.

22. Eiva Bernotiene, Gaby Palmer, Cem Gabay. The role of leptin in innate and adaptative immune responses. Arthritis Res Ther. 2006; 8(5): 217.

23. Bjorbaek C, Elmquist JK, Michl P, Ahima RS, van Bueren A, McCall AL, et al. Expression of leptin receptor isoforms in rat brain microvessels. Endocrinology. 1998; 139(8):3485-91. Epub 1998/07/29.

24. Myers MG. Jr. Leptin Keeps Working, Even in Obesity. Cell Metabolism Volume 21, Issue 6, 2 June 2015, Pages 791-792.

25. Bray MS, Loos RJ, McCaffery JM, Ling C, Franks PW, Weinstock GM, et al. NIH working group report-using genomic information to guide weight management: from universal to precision treatment. Obesity (Silver Spring) 2016; 24:14-22.

26. Heymsfiel SB, Gonzalez MC, Shen W, Redman L, Thomas D. Weight loss composition is one fourth fat-free mass: a critical review and critique of this widely cited rule. Obes Rev 2014; 15:310-21.

27. Bays HE. Adiposopathy is "sick fat" a cardiovascular disease? J Am Coll Cardiol. 2011; 57(25):2461-73

28. Brown NK, Zhou Z, Zhang J, Zeng R, Wu J, Eitzman DT, et al. Perivascular adipose tissue in vascular function and disease: A review of current research and animal models. Arterioscler Thromb Vasc Biol. 2014; 34:1621-30.

29. Wei Shen, ZiMian Wang, Mark Punyanita, Jianbo Lei, Ahmet Sinav, John G. Kral, et al Adipose tissue quantification by imaging methods: a pro-posed classification. Obes Res 2003; 11:5-16.

30. Grant RW, Dixit VD. Adipose tissue as an immunological organ. Obesity (Silver Spring) 2015; 23:512-8

31. Bays H. Adiposopathy, "sick fat," Ockham's razor, and resolution of the obesity paradox. Curr Atheroscler Rep. 2014; 16(5):409.

32. Farooqi IS, Jebb SA, Langmack G, Lawrence E, Cheetham CH, Prentice AM, et al. Effects of recombinant leptin therapy in a child with congenital leptin deficiency. N Engl J Med 1999; 341:879-884

33. Considine RV, Sinha MK, Heiman ML, Kriauciunas A, Stephens TW, Nyce MR, et al Serum immunoreactive-leptin concentrations in normal-weight and obese humans. N Engl J Med. 1996; 334(5):292-5.

34. Hall JE, da Silva AA, do Carmo JM, Dubinion J, Hamza S, Munusamy S, et al. Obesity induced hypertension: role of sympathetic nervous system, leptin, and melanocortins. J Biol Chem 2010; 285:17271-6.

35. Ferrannini E, Camastra S, Gastaldelli A, Maria Sironi A, Natali A, Muscelli E, et al Beta-cell function in obesity: ef- fects of weight loss. Diabetes 2004; 53:Suppl 3:S26 S33.

36. Wu J, Cohen P, Spiegelman BM. Adaptive thermogenesis in adipocytes: Is beige the new brown? Genes Dev. 2013; 27(3):234-50.

37. Tchkonia T, Thomou T, Zhu Y, Karagiannides I, Pothoulakis C, Jensen MD, et al. Mechanisms and metabolic implications of regional differences among fat depots. Cell Metab 2013; 17:644-56

38. Fitzgibbons TP, Czech MP. Epicardial and perivascular adipose tissues and their in fluence on cardiovascular disease: basic mechanisms and clinical associations. J Am Heart Assoc. 2014; 3(2):e000582.

39. Harms M, Seale P. Brown and beige fat: development, function and therapeutic po tential. Nature medicine. 2013. p. 1252-63.

40. McGown C, Birerdinc A, Younossi ZM. Adipose Tissue as an Endocrine Organ. Clin Liver Dis. 2014; 18(1):41-58

41. Padilla J, Jenkins NT, Vieira-Potter VJ, Laughlin MH. Divergent phenotype of rat thoracic and abdominal perivascular adipose tissues. Am J Physiol Regul Integr Comp Physiol. 2013; 304(7).

42. Crewe $\mathrm{C}, \mathrm{An}$ YA, et al. The ominous triad of adipose tissue dysfunction: inflammation, fibrosis, and impaired angiogenesis. J Clin Invest. 2017; 127 (1):74-82.

43. Schipper HS, Prakken B, Kalkhoven E, Boes M. Adipose tissue-resident immune cells key players in immunometabolism. Trends Endocrinol Metab 2012; 23(8):407-415.

44. Daniel A. Winer, Shawn Winer, Lei Shen, Persis P. Wadia Jason Yantha, Geoffrey Paltser, et al. B cells promote insulin resistance through modula-tion of T Cells and production of pathogenic IgG antibodies. Nat Med. 2011; 17(5):610-617.

45. Li J, Yu X, Pan W, Unger RH. Gene expression profile of rat adipose tis-sue at the onse of high fat-diet obesity. Am J Physiol Endocrinol Metab. 2002; 282(6): E1334-E1341.

46. Calle EE, Thun MJ. Obesity and cancer. Oncogene 2004; 23:6365-78.

47. Kaur J. A comprehensive review on metabolic syndrome. Cardiol Res Pract 2014; 2014:943162.

48. McCullough AJ. The clinical features, diagnosis and natural history of non- alcoholic fatty liver disease. Clin Liver Dis 2004; 8:521-33.

49. Kettner NM, Mayo SA, Hua J, Lee Ch, Moore DD, Fu L. Circadian Dys-function Induces Leptin Resistance in Mice. 2015, Cell Metabolism 22, 448-459.

50. Santillán Benítez JG, Mendieta Zerón H, Ordóñez Quiroz Á. La leptina en la carcinogénesis mamaria. Vías de señalización. Rev Química Viva. 2012;11(2):91-111.

51. Ernst MB, Wunderlich CM, Hess S, Paehler M, Mesaros A, Koralov SB, et al. Enhanced Stat3 activation in POMC neurons provokes negative feed-back inhibition of leptin and insulin signaling in obesity. J Neurosci. 2009 Sep 16; 29 (37): 11582-93.

52. Gong L, Yao F, Hockman K, Heng HH, Morton GJ, Takeda K,et al. Signal transducer and activator of transcription-3 is required in hypothalamic agouti- related protein/ neuropeptide Y neurons for normal energy homeostasis. Endocrinology. 2008; 149(7):3346-54.

53. Vásquez MEF. Señalización de la leptina. REB 25(2):50-4, 2006

54. Morrison CD. Leptin signaling in brain: A link between nutrition and cog-nition? Biochimica et Biophysica Acta. 2009; 1792(5):401-8.

55. The GBD obesity Collaborators, Health Effects of Overweight and Obesity in 195 Countries over 25 Years, N Engl J Med 2017; 377:13-27. 